Labor, and Welfare of Japan, as well as grants from the Ministry of Education, Culture, Sports, Science, and Technology of Japan.

Statement of Interest: None declared.

\section{REFERENCES}

1 Rosen SH, Castleman B, Liebow AA. Pulmonary alveolar proteinosis. N Engl J Med 1958; 258: 123-1143.

2 Kitamura T, Tanaka N, Watanabe J, et al. Idiopathic pulmonary alveolar proteinosis as an autoimmune disease with neutralizing antibody against granulocyte-macrophage colony stimulating factor. J Exp Med 1999; 190: 875-880.

3 Kitamura T, Uchida K, Tanaka N, et al. Serological diagnosis of idiopathic pulmonary alveolar proteinosis. Am J Respir Crit Care Med 2000; 162: 658-662.

4 Inoue $\mathrm{Y}$, Trapnell BC, Tazawa R, et al. Characteristics of a large cohort of autoimmune pulmonary alveolar proteinosis patients in Japan. Am J Respir Crit Care Med 2008; 177: 752-762.

5 Suzuki T, Sakagami T, Young LR, et al. Hereditary pulmonary alveolar proteinosis: pathogenesis, presentation, diagnosis, and therapy. Am J Respir Crit Care Med 2010; 182: 1292-1304.
6 Ishii H, Trapnell BC, Tazawa R, et al. Comparative study of highresolution CT findings between autoimmune and secondary pulmonary alveolar proteinosis. Chest 2009; 136: 1348-1355.

7 Ministry of Health, Labour and Welfare. Protecting personal identifiable data: ethical guidelines for epidemiological research Tokyo, Ministry of Health, Labour and Welfare of Japan, 2002; pp. 18-20.

8 Uchiyama M, Nagao T, Hattori A, et al. Pulmonary alveolar proteinosis in a patient with Behcet's disease. Respirology 2009; 14: 305-308.

9 Lederer DJ, Jelic S, Basner RC, et al. Circulating KL-6, a biomarker of lung injury, in obstructive sleep apnoea. Eur Respir J 2009; 33: 793-796.

10 Ohnishi T, Yamada G, Shijubo N, et al. Secondary pulmonary alveolar proteinosis associated with myelodysplastic syndrome. Intern Med 2003; 42: 187-190.

11 Pollack SM, Gutierrez G, Ascensao J. Pulmonary alveolar proteinosis with myeloproliferative syndrome with myelodysplasia: bronchoalveolar lavage reduces white blood cell count. Am J Hematol 2006; 81: 634-638.

12 Chung JH, Pipavath SJ, Myerson DH, et al. Secondary pulmonary alveolar proteinosis: a confusing and potentially serious complication of hematologic malignancy. J Thorac imaging 2009; 24: 115-118.

DOI: $10.1183 / 09031936.00092910$

\title{
Is NMR-based metabolomic analysis of exhaled breath condensate accurate?
}

\section{To the Editors:}

The metabolomic analysis of exhaled breath condensate (EBC) is a simple noninvasive approach for the study of respiratory system diseases. Previous studies introduced nuclear magnetic resonance (NMR)-based metabolomics as a method allowing a definite separation between healthy patients and patients with airway disease $[1,2]$. In these studies, the influence of external contaminants was also considered. DE LAURENTIIS et al. [1] reported that the removal of interfering residual external contaminants was crucial for correct EBC analysis and they proposed a cleaning protocol for the "complete removal of the disinfectant signals" from the reusable parts of the condensers.

In order to verify the influence of the disinfectant signals we have compared the EBC ${ }^{1} \mathrm{H}-\mathrm{NMR}$ spectra of a healthy subject obtained after the standard cleaning protocol (disinfected for 15 min using a $1.5 \%$ Descogen ${ }^{\mathrm{TM}}$ solution and flushed for 15 min with water) recommended by the manufacturer's guidelines and the International Consensus on EBC (fig. 1a) [3]; one that was obtained after the cleaning protocol proposed by DE LAURENTIIS et al. [1] (fig. 1b); and one obtained using a device without reusable condenser parts (fig. 1c). EBCs have been collected using an Anacon condenser (Biostec, Valencia, Spain). Sample collection and concentration ( $>99 \%$ water) were similar to those obtained in the procedure described by DE LAURENTIIS et al. [1] and a similar spectrum (fig. 1f) to the spectrum published by DE LAURENTIIS et al. [1] was obtained (fig. 1f, insert). Figure 1 shows that although many of the disinfectant signals present in the EBC spectrum have been removed by the DE LAURENTIIS et al. [1] cleaning protocol, there are some resistant peaks that are not observed in the spectrum in figure 1c. Furthermore, an EBC ${ }^{1} \mathrm{H}-\mathrm{NMR}$ spectrum of a healthy subject acquired using the device without reusable parts (fig. 1d) was compared with another control test (fig. 1e), collected in the same time scale using the condensation of room air pushed into the system by a continuous air spam device using flow and volumes similar to those typical of normal human respiratory function. In this case, it showed similar blank spectra with only two signals (lactate and an unidentified signal).

In our opinion, this comparison indicates that previous studies were not able to remove all disinfectant signals via the cleaning protocols. In addition, when the use of disinfectants was circumvented using a non-reusable device, we obtained blank spectra without metabolic information. We are persuaded to conclude that within the normal time periods used for EBC collection (10 min), NMR-based metabolomics does not have 


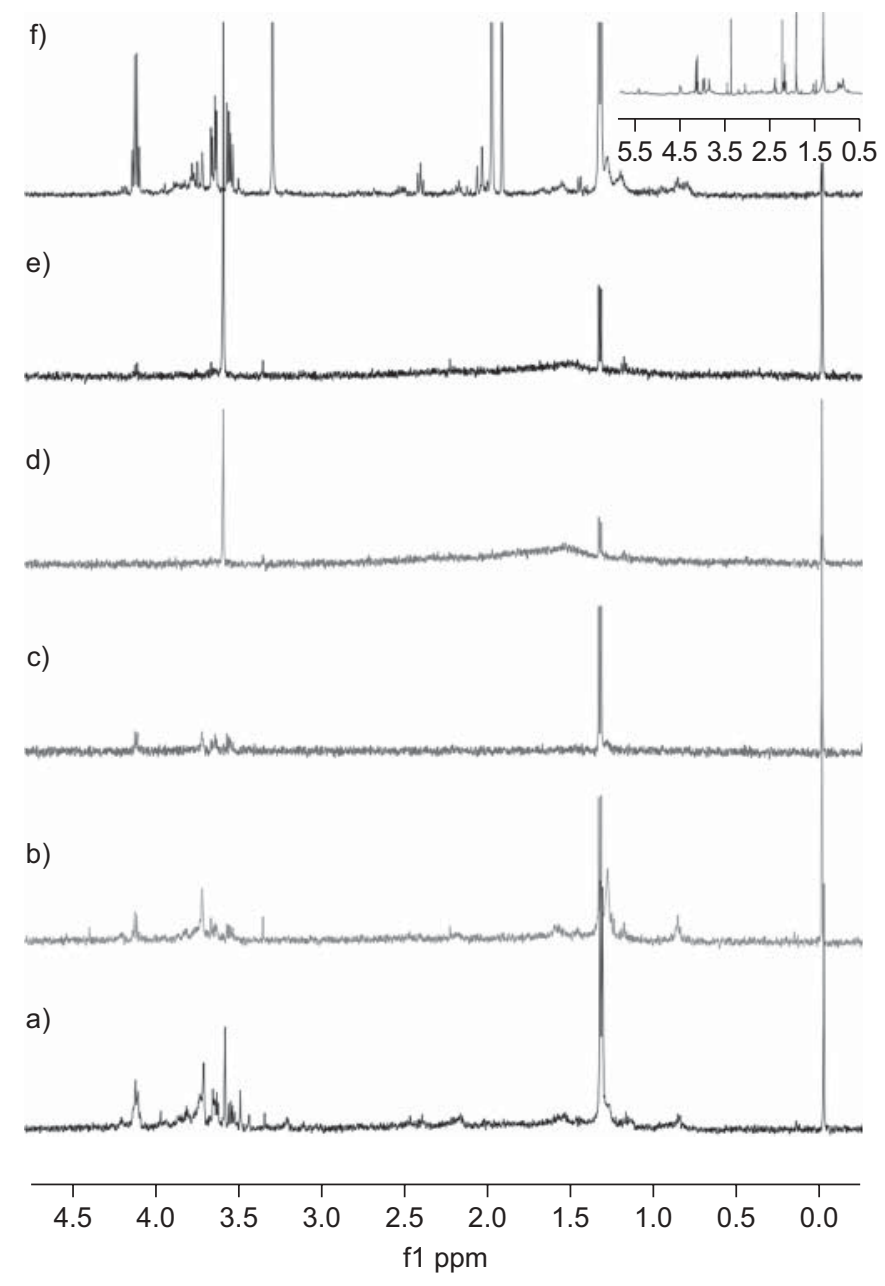

FIGURE 1. ${ }^{1} \mathrm{H}$-nuclear magnetic resonance (NMR) spectra of exhaled breath condensate (EBC) from a healthy subject after a) the standard cleaning protocol recommended by manufacturer's guidelines and the International Consensus on EBC; b) the cleaning protocol proposed by DE LAURENTIIS et al. [1]; c) and d) using a device without reusable condenser parts; and e) ${ }^{1} \mathrm{H}-\mathrm{NMR}$ spectra of the condensation of the room air pushed into the system by a continuous air spam device. EBC spectra a), b) and c) were collected from the same healthy subject and spectra c) and d) were collected in the same time scale. f) ${ }^{1} \mathrm{H}$-NMR spectrum of EBC with similar sample collection and disinfection to ${ }^{1} \mathrm{H}$-NMR spectrum of EBC published by DE LAURENTIIS et al. [1] (insert; reproduced from [1] with permission from the publisher). f1: first frequency dimension.

the degree of sensitivity needed to detect the lung metabolic fingerprint present in these samples. For this reason, we propose a mass spectrometry (MS) approach for the metabolomic analysis of EBC.

MS-based metabolomics, here using ultra-performance liquid chromatography coupled to a time-of-flight mass spectrometer (UPLC-TOF-MS) requires similar sample preparation with a high degree of sensitivity. Through this technique, we can detect the metabolic fingerprint of the EBC not detected by ${ }^{1} \mathrm{H}-\mathrm{NMR}$ spectroscopy. Figure 2 shows the UPLC-TOF mass matrix of an EBC from a healthy subject acquired using the non-reusable device. With the aim of validating the MS-based metabolomics as a discriminatory method between healthy
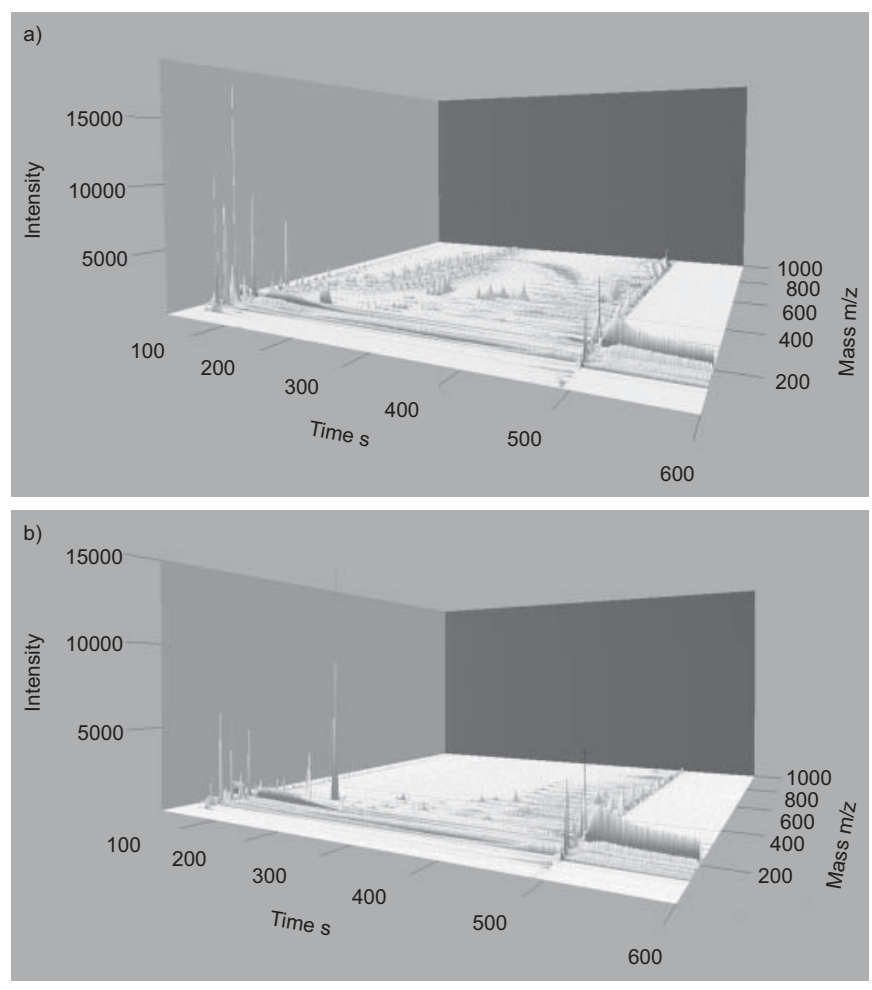

FIGURE 2. An ultra-performance liquid chromatography coupled to a time-offlight mass spectrometer three-dimensional mass matrix of an exhaled breath condensate from a) a healthy subject and b) a subject with chronic obstructive pulmonary disease, acquired using the non-reusable device. $\mathrm{m} / \mathrm{z}$ : mass to charge.

patients and patients with airway disease, preliminary data of a chronic obstructive pulmonary disease (COPD) metabolomic study are presented. A total of 28 EBC samples, 18 COPD and 10 healthy subjects were collected using the non-reusable device and the Anacon condenser. Lyophilised samples were diluted with $200 \mu \mathrm{L}$ of water and transferred into 96-well plates and maintained at $4{ }^{\circ} \mathrm{C}$ prior to injection of $5 \mu \mathrm{L}$ onto the column. Chromatographic separations were performed on a Acquity UPLC system (Waters Corp, Milford, MA, USA) using a 10 min gradient with mobile phase $\mathrm{A}$ of $0.1 \%$ formic acid (FA) in water and mobile phase B, $0.1 \%$ FA in acetonitrile. Electrospray (ESI) mass spectrometry experiments were performed on a Micromass LCT Premier (Waters) operated in positive ionisation mode. The raw data were processed using the open-source freeware XCMS (Scripps Center for Mass Spectrometry, La Jolla, CA, USA), which is able to detect and align metabolite peaks. Spectral differences between COPD and healthy subjects were verified by principal components analysis and partial least squares analysis as shown in figure 3.

In conclusion, the present study demonstrates that the cleaning protocols of the reusable condenser parts produce an artificial metabolic fingerprint not related to the endogenous metabolic pathway of the lungs. In addition, although NMR-based metabolomics have been successfully applied in the study of numerous biosamples (e.g. urine [4-6] and tissues [7]), this technique cannot be used to analyse EBC samples because the spectroscopic techniques do not have the sensitivity 

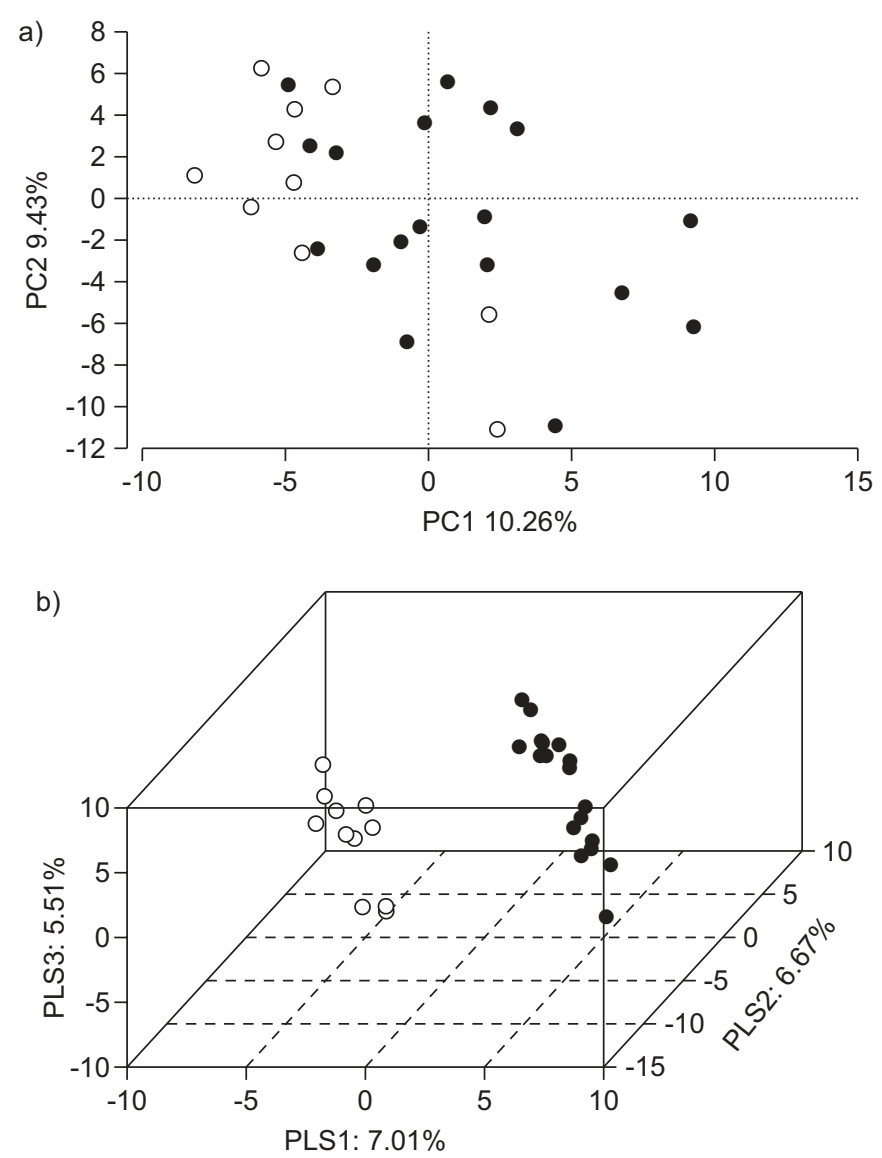

FIGURE 3. Multivariate analysis using ultra-performance liquid chromatography coupled to a time-of-flight mass spectrometer spectra of exhaled breath condensate from healthy and chronic obstructive pulmonary disease (COPD) subjects. a) Principal components analysis (PCA) score plot shows a separation between groups, which is clearly confirmed by b) partial least squares (PLS) analysis. $\bigcirc$ : control; $\bullet$ : COPD. PC1 and 2 are the first two principal components; PLS1 and 2 are the first two partial least squares.

required to observe the endogenous metabolites presented in the EBC. Finally, we propose UPLC-MS and the use of nonreusable devices as a standard metabolomic approach in the analysis of EBC.
J.L. Izquierdo-García*, G. Peces-Barba", S. Heili", R. Diaz E. Want ${ }^{+}$and J. Ruiz-Cabello*

${ }^{*}$ CIBERES, CIBER Enfermedades Respiratorias, Instituto de Estudios Biofuncionales, UCM, " CIBERES, CIBER Enfermedades Respiratorias, Fundación Jimenez DiazCAPIO, Madrid, 'Universitat Jaume I, Castellon, Spain, and ${ }^{+}$Imperial College London, London, UK.

Correspondence: J. Ruiz-Cabello, Instituto de Estudios Biofuncionales, Universidad Complutense Madrid, Paseo Juan XXIII, 1 Madrid 28040, Spain. E-mail: ruizcabe@farm. ucm.es

Support Statement: Funding was provided by the Spanish Ministry of Science and Innovation (SAF2008-05412), the Community of Madrid (S505-AGR-187) and EU under grant agreement ITN-FP7-264864.

Statement of Interest: None declared.

\section{REFERENCES}

1 de Laurentiis G, Paris D, Melck D, et al. Metabonomic analysis of exhaled breath condensate in adults by nuclear magnetic resonance spectroscopy. Eur Respir J 2008; 32: 1175-1183.

2 Carraro S, Rezzi S, Reniero F, et al. Metabolomics applied to exhaled breath condensate in childhood asthma. Am J Respir Crit Care Med 2007; 175: 986-990.

3 Horvath I, Hunt J, Barnes PJ, et al. Exhaled breath condensate: methodological recommendations and unresolved questions. Eur Respir J 2005; 26: 523-548.

4 Slupsky CM, Rankin KN, Fu H, et al. Pneumococcal pneumonia: potential for diagnosis through a urinary metabolic profile. J Proteome Res 2009; 8: 5550-5558.

5 Saude EJ, Obiefuna IP, Somorjai RL, et al. Metabolomic biomarkers in a model of asthma exacerbation: urine nuclear magnetic resonance. Am J Respir Crit Care Med 2009; 179: 25-34.

6 McClay JL, Adkins DE, Isern NG, et al. ${ }^{1} \mathrm{H}$ nuclear magnetic resonance metabolomics analysis identifies novel urinary biomarkers for lung function. J Proteome Res 2010; 9: 3083-3090.

7 Izquierdo-García JL, del Puerto-Nevado L, Peces-Barba G, et al. A metabonomic approach to evaluate COPD in a model of cigarette smoke exposure in mice. Metabolomics 2010; 6: 564-573.

DOI: $10.1183 / 09031936.00094010$

\section{Hypercalcaemia in asymptomatic sarcoidosis unmasked by a vitamin $\mathrm{D}$ loading dose}

\section{To the Editors:}

The risk of occurrence of hypercalcaemia induced by vitamin $\mathrm{D}$ in certain conditions has recently been summarised by KALLAs et al. [1]. Despite the high prevalence of vitamin D deficiency among the healthy population and observational associations with cardiovascular disease, autoimmune diseases, some types of cancer, tuberculosis and mortality [2, 3], there are currently no data to justify widespread use of vitamin D supplementation, taking into account the lack of large prospective randomised controlled trials.

We would like to share our experience with calcitriol-mediated hypercalcaemia in an apparently healthy individual. A 26-yrold obese female with a body mass index of $48.4 \mathrm{~kg} \cdot \mathrm{m}^{-2}$ was transferred to the endocrinology outpatient clinic of the 